\title{
Atención primaria social en Colombia: una mirada desde una experiencia exitosa
}

\section{Social primary care in Colombia: A look from a successful experience}

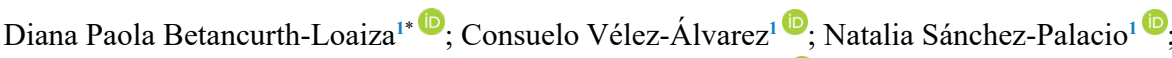 \\ Claudia Patricia Jaramillo-Ángel ${ }^{1}$ (1) \\ *diana.betancurth@ucaldas.edu.co
}

Forma de citar: Betancurth-Loaiza DP, Vélez-Álvarez C, Sánchez Palacio N, Jaramillo-Ángel CP. Atención Primaria Social en Colombia: una mirada desde una experiencia exitosa. Salud UIS. 2022; e22016. doi: https://doi.org/10.18273/saluduis.54.e:22016 (c) (i)

\section{Resumen}

Introducción: la estrategia de Atención Primaria Social ha sido valorada por la Organización Panamericana de la Salud como una experiencia exitosa. Objetivo: analizar la experiencia de Atención Primaria Social en el departamento de Caldas en el marco de un proceso de sistematización. Materiales y métodos: estudio de sistematización de experiencias, las unidades de análisis y de trabajo fueron documentos municipales y departamentales, información del Observatorio Social de Caldas y relatos de diferentes actores. Técnicas: 71 entrevistas a profundidad a informantes claves, 27 grupos focales, 11 diarios de campo y revisión documental. La información fue recolectada en 6 fases y luego analizada en el software ATLAS.ti 8.0. Resultados: la estrategia tuvo un recorrido histórico bajo lineamientos nacionales e internacionales desde los años 80 hasta la actualidad, fue modificada en 2013 al articular actores y sectores público-privados para convertirla en "Atención Primaria Social”. El modelo operativo se cimienta en la transectorialidad y educación interdisciplinar, bienestar y salud familiar, y participación social y comunitaria, cuyo eje central son los actores, familias y comunidades. Se destacan actividades como caracterización familiar, intervenciones a familias priorizadas y unidades de análisis para apoyo, monitoreo y seguimiento. Conclusiones: han participado diferentes actores en la comprensión de la mirada y el abordaje de la salud desde lo "social", un actor relevante ha sido la comunidad, acompañada de sus líderes y la academia. La voluntad política del gobierno nacional, departamental y municipal es relevante para el éxito de la estrategia. Los indicadores en salud han sido impactados por el avance en la implementación de la estrategia de Atención Primaria Social.

Palabras clave: Atención primaria de salud; Prácticas interdisciplinarias; Aprendizaje social; Salud pública; Participación de la comunidad; Determinantes sociales de la salud.

\footnotetext{
${ }^{1}$ Universidad de Caldas. Manizales, Colombia. 


\begin{abstract}
Introduction: The Social Primary Care strategy has been evaluated by the Pan American Health Organization as a successful experience. Objective: To analyze the experience of Primary Social Care in the department of Caldas within the framework of a systematization process. Methods: Systematization study of experiences, the units of analysis and work were municipal and departmental documents, information from the Social Observatory of Caldas and accounts of different actors. Techniques: 71 in-depth interviews with key informants, 27 focus groups, 11 field diaries and documentary review, the information was collected in 6 phases and then analyzed in ATLAS. ti 8.0 software. Results: The strategy had a historical trajectory under national and international guidelines from the 1980s to the present, it was modified in 2013 by articulating actors and public-private sectors to turn it into "Social Primary Care". The operational model is based on trans-sectoriality and interdisciplinary education, family wellbeing and health, and social and community participation, whose central axis are the actors, families, and communities. Activities include family characterization, interventions for prioritized families and units of analysis for support, monitoring and follow-up. Conclusions: Different actors have participated in the understanding of the "social" approach to health, a relevant actor has been the community accompanied by its leaders and academia. The political will of the national, departmental and municipal governments is relevant for the success of the strategy. Health indicators have been impacted by the progress in the implementation of the Social Primary Health Care strategy.
\end{abstract}

Keywords: Primary health care; Interdisciplinary placement; Social learning; Public health; Community participation; Social determinants of health.

\section{Introducción}

La Declaración de Alma Ata presentó la estrategia de Atención Primaria en Salud (APS) como la mejor manera de dar salud a los pueblos ${ }^{1}$. Por su parte, la Organización Panamericana de la Salud (OPS) propuso un plan de Renovación de la APS basado en la equidad y en la solidaridad, cuyo propósito fundamental fue revitalizar la capacidad de los países para resolver los problemas de salud existentes y a sus retos en esta materia ${ }^{2}$. Para esto se dará cuenta de una experiencia exitosa desarrollada en el departamento de Caldas, así como de algunas consideraciones sobre los modelos que en Colombia han antecedido a estas nuevas apuestas que, desde la atención en salud, se centran en lo comunitario y social del contexto.

En Colombia con la Ley 1438 de 2011, se redefinió la APS como una estrategia de coordinación intersectorial que permite la atención integral e integrada desde la salud pública, la promoción de la salud, la prevención de la enfermedad, el diagnóstico, el tratamiento y la rehabilitación en todos los niveles de complejidad a fin de garantizar un mayor nivel de bienestar en los usuarios ${ }^{3}$. En la actualidad, se adopta el Modelo de Acción Integral Territorial (MAITE) ${ }^{4}$, por el cual se plantea un marco estratégico fundamentado en "la Atención Primaria en Salud, con perspectiva de salud familiar y comunitaria, el cuidado, la gestión integral del riesgo y el enfoque diferencial para los distintos territorios y poblaciones", con el fin de promover acciones que faciliten la gestión de la salud pública, articulada con una adecuada prestación de los servicios en cada territorio.

Estos planteamientos promueven un viraje de la APS hacia un modelo centrado en la participación social, en la cual los trabajadores de la salud y actores sociales gestionen y promuevan acciones convenientes a las necesidades de la comunidad y al derecho a la salud a partir de una atención primaria eminentemente social $^{5}$. Sin duda, los sistemas de salud que fortalecen esta estrategia logran mejores resultados por tener una estructura más inclusiva, eficaz y efectiva para el mejoramiento de la salud física y mental de las personas, reflejándose en un alto impacto en el desarrollo social y comunitario.

Es así como las transformaciones que ha sufrido la Atención Primaria en Salud en el contexto de Caldas, departamento de la subregión andina del Eje Cafetero de Colombia, han permitido concentrar los esfuerzos en aquellos municipios más rezagados, al establecer planes y metas encaminados a reducir las brechas sociales y mejorar las condiciones de vida mediante un modelo operativo y participativo genuino. En este sentido, la estrategia Atención Primaria Social (APS) ha permitido realizar diagnósticos y caracterizaciones de las familias, así como promover procesos educativos, de planeación y ejecución de rutas de atención integrales que articulan lo comunitario, la promoción del autocuidado y el seguimiento ${ }^{6}$, todo esto centrado en los actores sociales y particularmente sus circunstancias de vida. 
El impacto se ha visto reflejado en los resultados alcanzados, que le permitieron al Departamento de Caldas ser elegido como el mejor en indicadores de salud en $2019^{7}$, al evaluarse cinco dimensiones: percepción de los usuarios, financiamiento de la salud, desempeño institucional, participación ciudadana y gobernanza. Esta multidimensionalidad posibilitó al Departamento no solo situarse como ejemplo de un sistema bien gestionado, sino también abrir nuevos caminos, tanto de investigación como de ordenación de los recursos humanos y no humanos partícipes.

Por lo anterior, la OPS catalogó la estrategia como un legado que Caldas le entregaba al mundo, como un modelo con las capacidades de abarcar los desafíos a los cuales se ve sometido producto de las particularidades de los contextos. Es por esto y por otros motivos que surge la necesidad y relevancia de analizar el proceso y los resultados de cara a la posibilidad de brindar recomendaciones para la toma de decisiones y trazados políticos en el contexto local, nacional e internacional ${ }^{8}$. Este trabajo amplía elementos conceptuales y metodológicos que aportan a la necesidad de hacer evidente la articulación entre actores y sectores para fortalecer las redes integradas de servicios de salud en el marco de la atención primaria9.

Experiencias como la de Caldas se vivencian en el país, no obstante, no siempre se accede a la manera en que se desarrollan las estrategias de forma sistemática, como punto de partida para nuevos procesos y así visibilizar la unión de la teoría con la praxis, es decir, dar a conocer todo lo que aconteció durante su desarrollo, entender por qué se obtuvieron los resultados documentados $\mathrm{y}$ abstraer aquellos aspectos que permitieron realizar mejoras para futuras acciones ${ }^{10}$. Los resultados que se presentan son aportes para que a nivel nacional se valore de manera significativa el impacto que tiene la APS en la dinamización de la Política de Atención Integral como marco estratégico, y del modelo mismo en pro de los indicadores de salud y la calidad de vida de la población ${ }^{11}$.

Para finalizar, se debe plantear que la sistematización requiere de una metodología estructurada, en la que se tengan en cuenta los éxitos y fracasos de la experiencia, es lo que permite que la práctica realizada se pueda mejorar y de esta manera ser interiorizada y adaptada por otros ${ }^{12}$.

El objetivo del trabajo desarrollado fue analizar la experiencia de Atención Primaria Social en el Departamento de Caldas en el marco de un proceso de sistematización.
Como objetivos específicos que orientan el desarrollo de este trabajo están:

- Analizar el contexto histórico situación inicial y los elementos del contexto que dieron origen a la implementación de la estrategia de Atención Primaria Social en el departamento de Caldas.

- Describir el modelo operativo de la Atención Primaria Social en el departamento de Caldas.

- Describir la Atención Primaria Social en el Departamento de Caldas desde la voz de las comunidades.

\section{Materiales y métodos}

Se realizó un estudio de sistematización de experiencias basado en los lineamientos de la $\mathrm{FAO}^{13}$, a partir de lecciones aprendidas e iniciativas de políticas públicas entre el 2016 y 2019 en el departamento de Caldas, Colombia.

La sistematización de experiencias como tipo de estudio es entendido como "aquel proceso de ordenamiento y clasificación de datos e informaciones, en el que estructurando de manera precisa categorías y relaciones, se posibilita la constitución de bases de datos organizadas", en este sentido, tiene como propósito exponer y explicar los cambios sucedidos en la realización de un proyecto o implementación de una estrategia, así como los factores intervinientes, los resultados y las lecciones que se aprendieron en el camino $^{13}$. Este procura porque los diferentes actores sociales se involucren en la generación de nuevo conocimiento, en la construcción de procesos de aprendizaje e ideas de proyectos a partir de experiencias, datos e información documentada ${ }^{10}$.

La sistematización busca describir y comprender todo lo que aconteció durante el desarrollo de una experiencia, es decir, entender por qué se obtuvieron los resultados documentados y abstraer aquellos aspectos que permitan realizar procesos de mejora para futuras acciones $^{10}$.

Es así como sistematizar implica hacer un proceso iterativo, en donde se tengan en cuenta los éxitos y fracasos de la experiencia, pues gracias a este enfoque la práctica realizada se puede mejorar y de esta manera ser adaptada por otros ${ }^{12}$.

Como unidad de trabajo y de análisis, se tuvieron en cuenta los documentos derivados del trabajo en APS de los 27 municipios del Departamento, la información 
proveniente del Observatorio Social de Caldas, así como también de los diferentes sectores (académicos, empresariales, cultuales, sociales, entre otros) y actores, tanto comunitarios como gubernamentales. Se empleó una herramienta creada por la Dirección Territorial de Caldas, que permite la interoperabilidad de datos, el uso de instrumentos de información familiar y el monitoreo de la dinámica familiar para la generación de las respuestas que el estado puede dar a las demandas de la sociedad.

El equipo estuvo integrado por tres investigadores principales, un coordinador general, un asesor experto y 6 auxiliares de investigación. Estas últimas, se seleccionaron teniendo en cuenta dos criterios básicos: que tuviera nivel de formación profesional con experiencia relacionada con el objeto de estudio y residieran mínimo 6 meses en el municipio de la subregión donde trabajarían.

Respecto al procedimiento, este se realizó en 6 fases, así:

Fase 0, inicio del proceso: se llevó a cabo el contacto inicial con los actores de los municipios para coordinar las actividades, se realizó la asignación de funciones del equipo, además de desarrollar las correspondientes capacitaciones de las auxiliares para la recolección de la información por las 6 subregiones, trabajo que fue auditado durante todo el proceso de trabajo de campo. Este fue auditado diariamente durante el trabajo de campo.

Fase 1, actores del proceso de desarrollo: se realizó de forma individual y grupal a través de 71 entrevistas a profundidad y 27 grupos focales, en los que participaron aquellos actores que tenían relación directa con la experiencia y, en algunos casos, también se tuvieron en cuenta actores indirectos de los municipios.

Fase 2, situación inicial y sus elementos de contexto: se partió de la descripción del problema o la oportunidad a la que está referida la experiencia de Atención Primaria Social en Caldas, a través de la revisión documental y de la realización de entrevistas a profundidad.

Fase 3, proceso de intervención y sus elementos de contexto: se tuvieron en cuenta las actividades que constituyeron el proceso, secuencia en el tiempo, rol de cada uno de los principales actores, métodos o estrategias empleadas, medios y recursos utilizados para las diferentes actividades y los factores del contexto que facilitaron o dificultaron el desarrollo del trabajo.
La información recolectada en campo se consignó en 11 diarios de campo que luego fueron complementados con la transcripción de las entrevistas a profundidad.

Fase 4, situación final y sus elementos de contexto: fueron descritos los resultados de la experiencia al momento de hacer la sistematización y se consideraron los resultados tangibles e intangibles, los cuales constituyeron un valioso recurso para el desarrollo futuro de las comunidades locales.

Fase 5, lecciones aprendidas de la sistematización: estas permitieron generar nuevos conocimientos, aportes, iniciativas de mejoramiento, y obtener pautas para optimizar la capacidad de toma de decisiones de los agentes encargados del desarrollo local. Se realizó la validación y retroalimentación de los resultados con los actores responsables de la estrategia a nivel departamental. Finalmente, se comunicaron los resultados por medio de un informe final y se organizó un encuentro de socialización con los actores principales del proceso tanto de los municipios como del departamento, se contó con la participación de representantes de la Organización Panamericana de la Salud.

La información fue analizada y procesada en el programa Atlas-Ti versión 8.0, en el que emergieron 5 categorías y 14 subcategorías. (Figura 1)

El estudio cumplió con los planteamientos éticos de la Resolución 008430 de 1993, artículo $11^{14}$, en el que se consideró como "investigación sin riesgo" y se tuvo en cuenta en todo el proceso el diligenciamiento del consentimiento informado por parte de los participantes.

\section{Resultados}

Los resultados serán presentados en función de tres categorías macro de análisis sobre las cuales fueron estructurados los objetivos específicos de la investigación: construyendo historia en Caldas, modelo operativo de Atención Primaria Social y la experiencia de APS desde la voz de las comunidades.

\section{Construyendo historia en Caldas}

Desde 1980 en Caldas se inició la implementación de la estrategia Atención Primaria en Salud, fundamentada en planes y programas de promoción de la salud rural y operativizada a través de Unidades Primarias de Atención (UPA), las cuales se encontraban bajo el liderazgo de las instituciones hospitalarias municipales. 


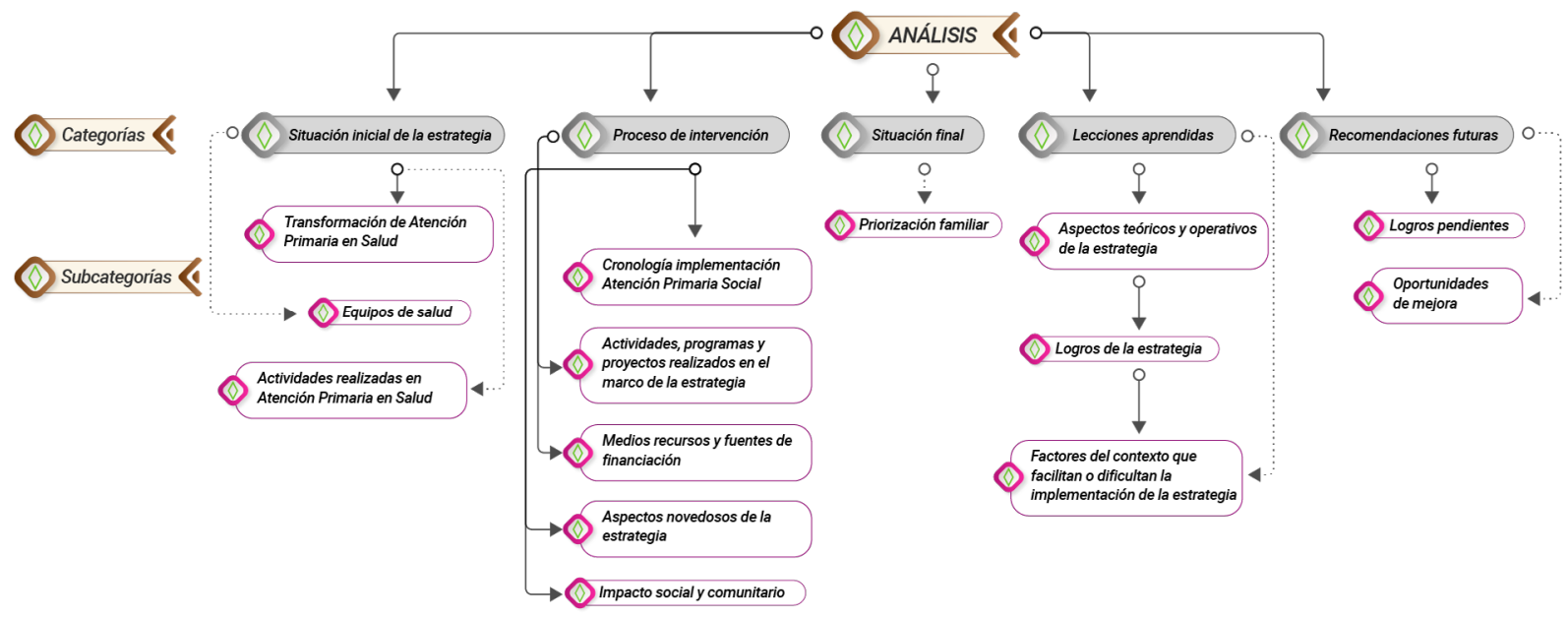

Figura 1. Categorías y subcategorías de investigación Fuente: Elaboración propia

El equipo de salud rural se conformaba por un médico, una enfermera, un odontólogo y un promotor de saneamiento, quienes se desplazaban periódicamente a las zonas para realizar acompañamiento a las familias campesinas en programas de salud pública, promoción de la salud y control de la enfermedad. Fue en el marco de este proceso que se construyó la ficha familiar, en la que se registraban todas las variables de interés en salud pública, detección temprana, protección específica y saneamiento. Con el análisis de las fichas se identificaba el riesgo familiar y se realizaban visitas domiciliarias de acompañamiento y capacitación desde las UPA para superar y evitar los riesgos.

Debido a los cambios normativos derivados de la ley 100 de $1993^{15}$, que reglamentó el nuevo Sistema General de Seguridad Social en Salud (SGSSS), la APS se debilitó, y fue hasta el año 2001 que en el municipio de La Dorada (departamento de Caldas) se retomó la estrategia bajo el nombre de "Atención Primaria Social" (APS), en tanto se vincularon otras áreas como educación, cultura, deporte y turismo, con el fin de brindar una oferta más integral a la comunidad, que incluía valoración de la pobreza, desigualdad e inequidad. Bajo esta estrategia, se continuó con la caracterización familiar por medio de la ficha, se diseñó un observatorio en el cual se sistematizó la información familiar y se consolidaron las Células de Acción Solidaria, que funcionaban como unidad, o puesto de acciones intersectoriales donde se realizaba atención en salud, brigadas intersectoriales, jornadas barriales, entre otros.

Estas acciones significaron un mejoramiento de los indicadores de salud municipales reflejados en experiencias positivas del municipio, entre las que se destacaron disminución de la mortalidad materna y los casos de dengue. Para el año 2013, con el cambio de gobierno nacional, se apropió la estrategia en todo el departamento, con la intención de consolidar un modelo integral y multidimensional. (Figura 2).

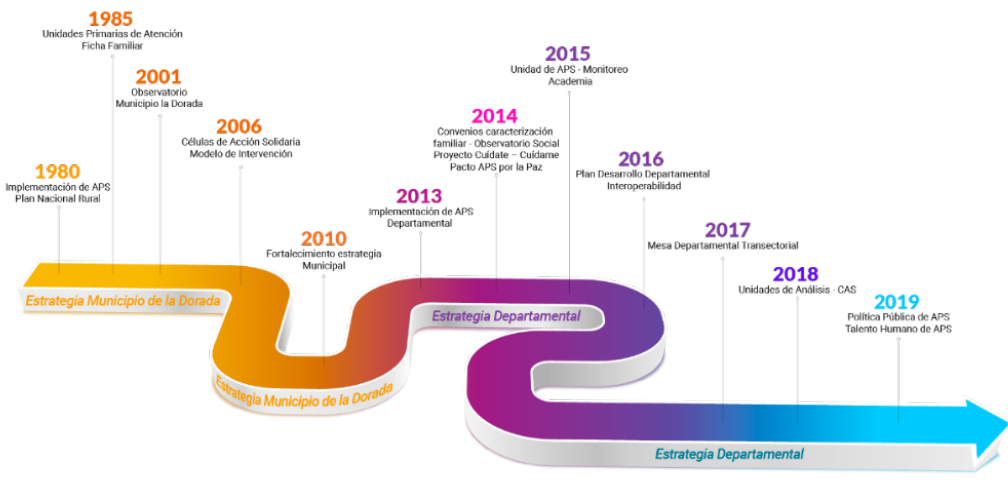

Figura 2. Contexto histórico de Atención Primaria Social en el Departamento de Caldas Fuente: Elaboración propia 


\section{Caldas: modelo operativo de Atención Primaria} Social

En la Figura 3 se presenta el modelo operativo de la estrategia, el cual es el resultado del análisis integral realizado durante la sistematización de la experiencia.
En él se consolidan y articulan todos los elementos que dan cuenta del proceso realizado en el departamento, además de aquellos que son transversales para favorecer el dinamismo y desarrollo desde lo social y desde lo comunitario.

\section{Modelo operativo de Atencion Primaria Social en Caldas}

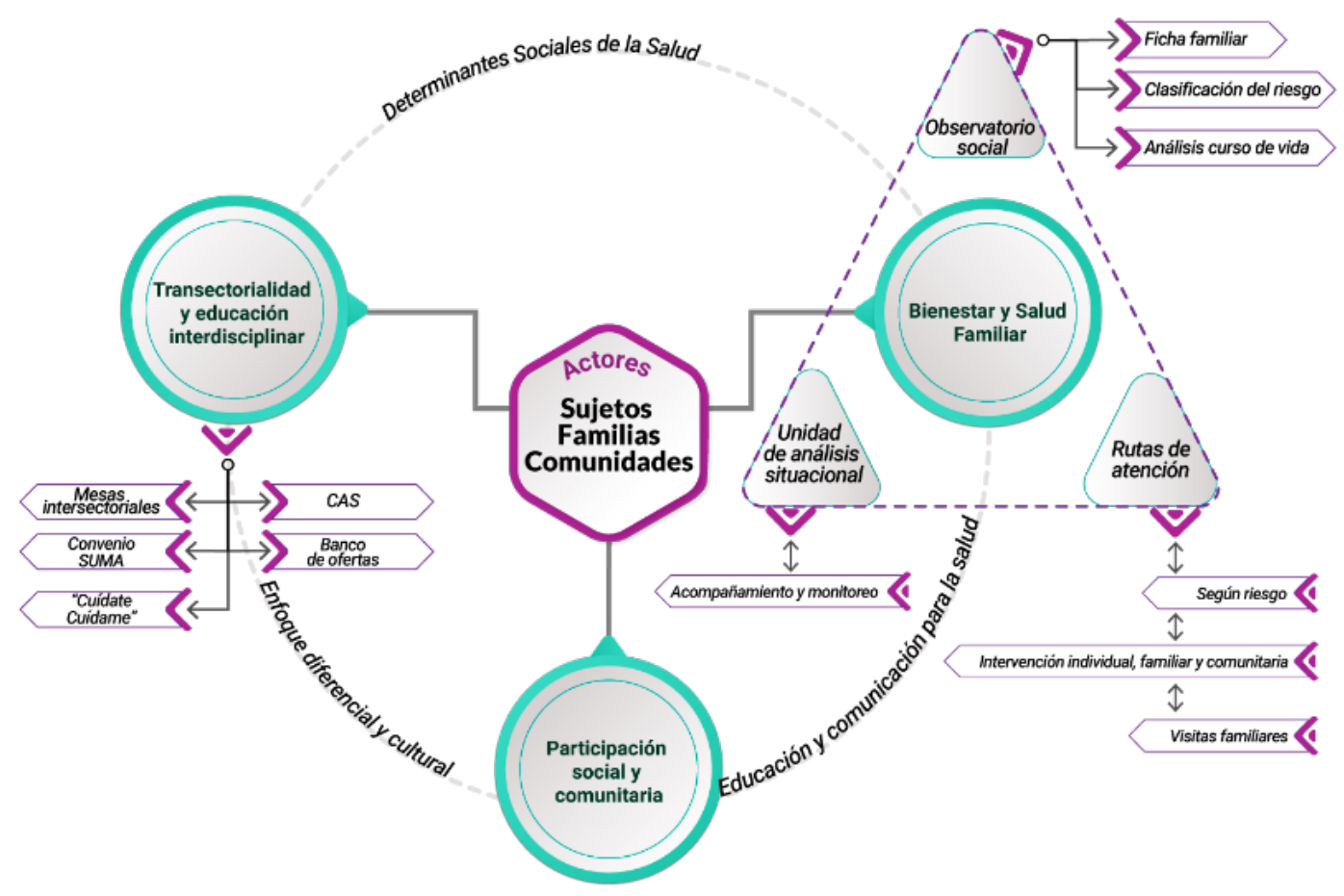

Figura 3. Modelo Operativo de Atención Primaria Social en Caldas

Fuente: Elaboración propia

\section{Transectorialidad y educación interdisciplinar}

En Caldas se incluyeron la transectorialidad y educación interdisciplinar como elementos esenciales para impactar la comunidad. Esto se realizó a través de la conformación de:

- Mesas intersectoriales departamentales y municipales: radicadas mediante actos administrativos desde 2017 e integradas por la academia, las alcaldías municipales, los hospitales y actores de diferentes áreas disciplinares. Las mesas se realizan trimestralmente, con el objetivo de priorizar, planificar y ejecutar procesos de APS en el departamento.

- Centros de Atención Social (CAS): se configuran en espacios sociales dinámicos (físicos o itinerantes), con una estructura que favorece el acercamiento de las ofertas institucionales a la comunidad.
En el momento del estudio se contaba con más de 50 territorios CAS ubicados en las zonas del departamento con mayor riesgo.

- Bancodeofertasintersectoriales:repositoriopúblico en el que se sistematiza el portafolio de servicios de los entes municipales y departamentales, tanto públicos como privados, dirigidos a la comunidad.

- Convenio Sistema Universitario de Manizales (SUMA): alianza creada para fortalecer la vinculación de la academia en las actividades de APS por medio de prácticas académicas universitarias.

- Proyecto Cuídate-Cuídame: creado en 2013, busca promover el autocuidado, los hábitos y estilos de vida saludables y forjar actitudes positivas en los ciudadanos del departamento. 


\section{Bienestar y salud familiar}

Con este eje se busca impactar el sujeto, su familia y la comunidad a través de estrategias como:

- Observatorio Social de Caldas: Sistema integrador de la información del departamento, que posibilita identificar las necesidades de la población y la dinámica del riesgo familiar a través de la interoperabilidad de datos, sistemas de georreferenciación y procesamiento estadístico predictivo. Utiliza como instrumento de recolección de información la ficha familiar, implementado desde las entidades municipales para conocer el estado de las familias basado en Determinantes Sociales de Salud (DSS).

- Rutas de Atención: programas de acción y actividades realizadas a través de visitas domiciliarias, jornadas de salud y bazares. Pueden estar dirigidas al individuo, a la familia o la comunidad en función del nivel de riesgo identificado.

- Unidad de Análisis Situacional: espacios municipales y del departamento para la búsqueda de soluciones, toma de decisiones y evaluación en lo relacionado con el abordaje de los riesgos sociales. Se realizan bimestralmente con la participación de entes públicos y privados que están al servicio de la comunidad.

\section{Participación social y comunitaria}

Desde el departamento se ha buscado dinamizar la participación social y comunitaria por medio de metodologías como el aprendizaje experiencial y la Educación y Comunicación para la Salud $(\mathrm{ECpS})^{16}$. En virtud de ello, brinda asistencia técnica a los municipios en lo relacionado con los mecanismos para socializar la estrategia de APS, conformar comités de participación comunitaria, capacitar en derechos y deberes, constituir mesas de concertación con trabajo intercultural, entre otros. La implementación de las acciones municipales es evaluada anualmente desde la administración departamental como parte del seguimiento y monitoreo a la gestión pública, siendo este elemento el más débil del modelo operativo.

\section{La experiencia de APS desde la voz de las comunidades}

La realización de grupos focales y entrevistas en los municipios permitió reconocer las características particulares sobre las cuales se fundamenta la APS. La Figura 4 evidencia cómo los actores reconocen la cronología de la APS y la manera en que las instituciones municipales y departamentales han aportado a la construcción de un modelo operativo centrado en lo social y lo comunitario. En este se resaltan los procesos de caracterización familiar, unidades de análisis y CAS basados en la intersectorialidad y los DSS.

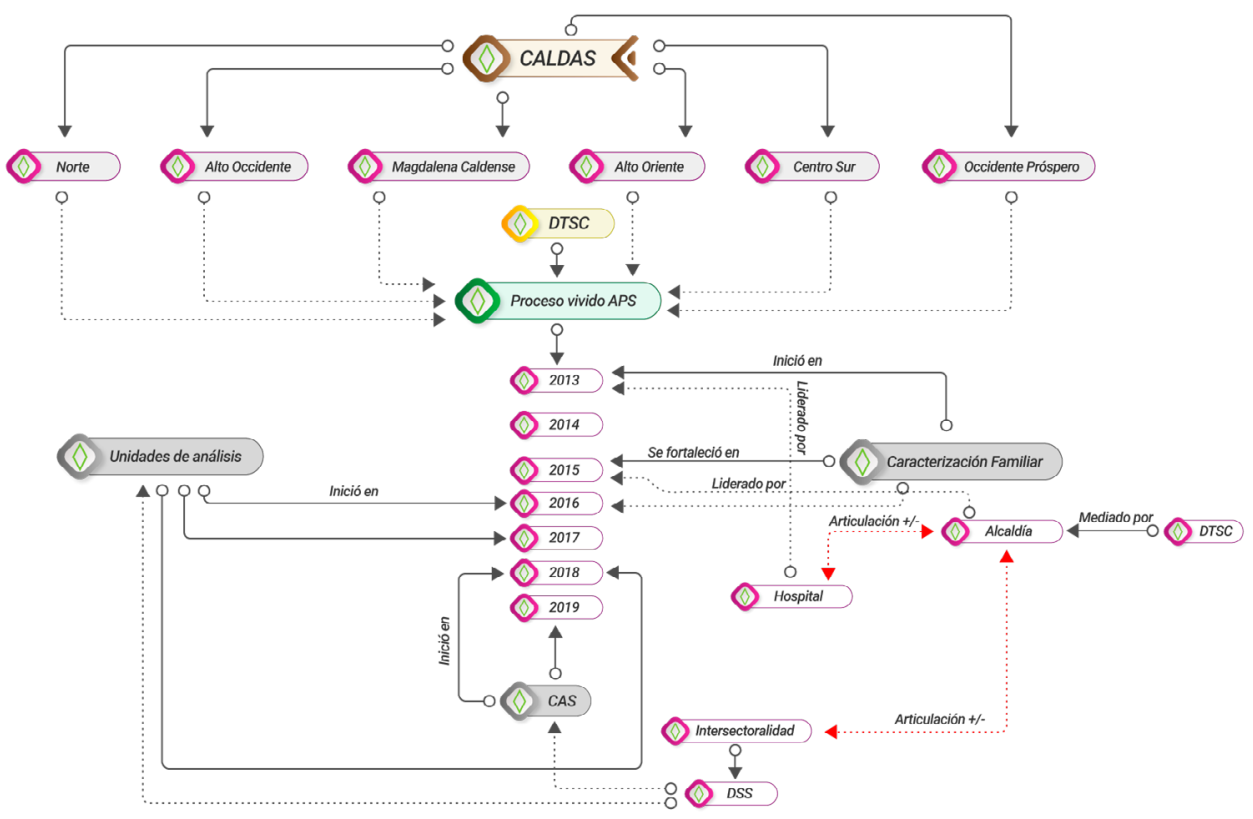

Figura 4. Red de análisis proceso vivido APS Fuente: Elaboración propia 
Las comunidades resaltan la presencia activa de las administraciones departamentales y municipales en la construcción de su territorio, además de la participación de la academia en la implementación de programas y proyectos construidos con y para la comunidad (Figura 5). Entre ellos se destacan:
- Programas: ambientales, juveniles, escolares, agrícolas, veterinarios e interculturales.

- Proyectos: huertas, cocinas ecológicas, emprendimiento, mercados campesinos, educación continuada, alfabetización, promoción de la salud, envejecimiento saludable y reciclaje.

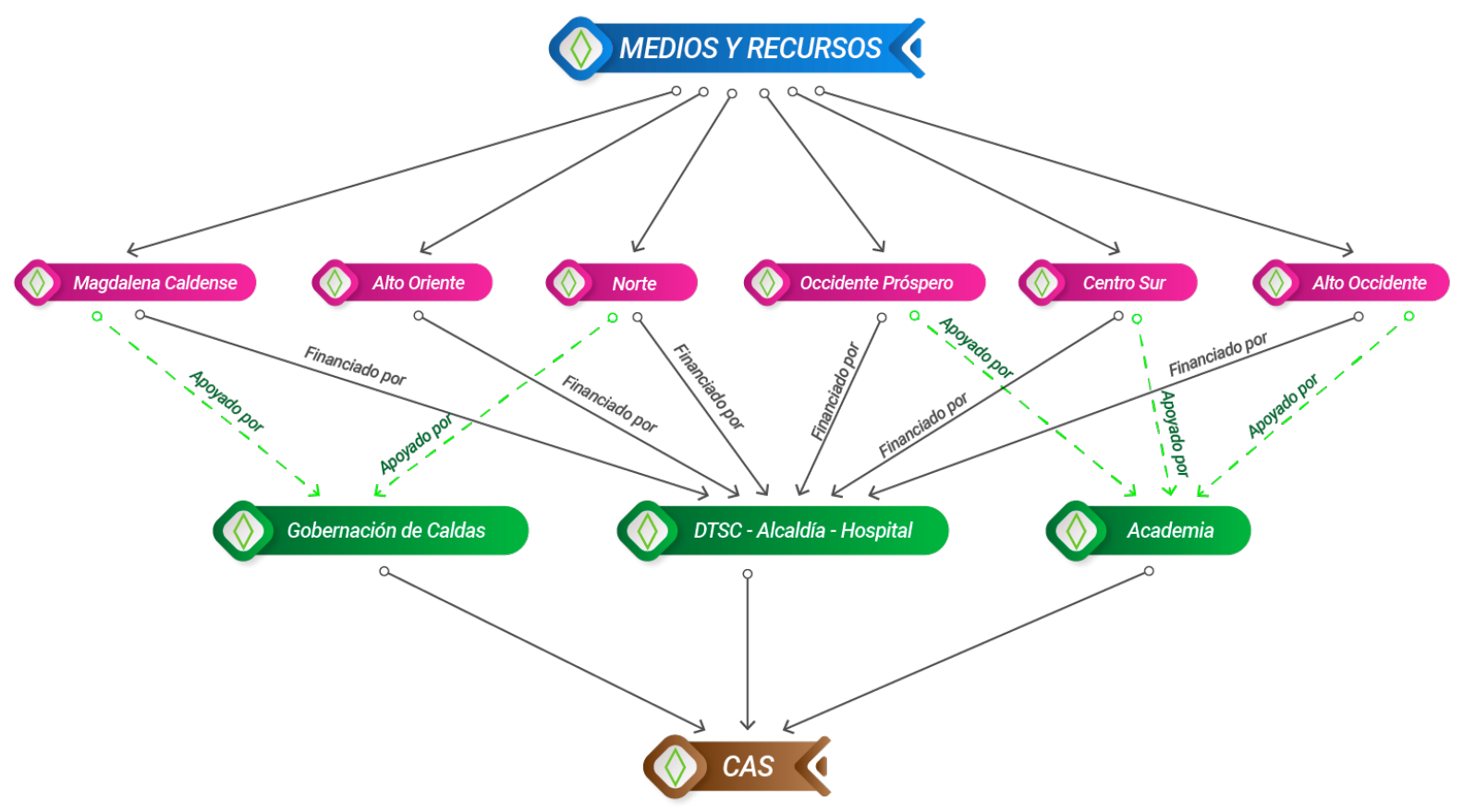

Figura 5. Medios y recursos de APS

Fuente: Elaboración propia

\section{Discusión}

Los resultados del presente estudio corroboran los esfuerzos a través de los años por mantener vigente la APS en los pueblos, 40 años después de la declaración de Alma Ata (1978) $)^{17}$. Tasca et al. ${ }^{18}$ indican que en la actualidad los países enfrentan grandes desafíos en cuanto a fortalecer lineamientos para la gobernanza, rectoría, acuerdos regionales y en la manera de desarrollar el modelo más adecuado con recomendaciones claves según los contextos sociales ${ }^{19}$.

Los resultados evidencian cómo en cada municipio el modelo ofrece elementos para describir en el tiempo los aspectos comunes, divergencias, limitantes, logros y lecciones aprendidas, y develan las bases fundamentales para la generación de nuevos procesos y formulación de políticas públicas a futuro, en aspectos como bienestar, participación social, entre otros ${ }^{20}$.
Colombia ha tenido diferentes experiencias en relación con el análisis histórico de la $\mathrm{APS}^{20-22}$, cuyo hallazgo en común también coincide con el presente estudio en la importancia de armonizar la estrategia con el actual sistema de salud, lo que debe ir acompañado de evaluaciones de impacto, de modo que se visibilice la superación de las brechas de inequidad por medio del análisis de los determinantes sociales. Esto precisa de una vinculación estrecha con lineamientos de justicia social e inclusive territorial, más que con la esencia asistencialista y biomédica del sector salud, lo que explica la importancia de una nueva denominación, la "Atención Primaria Social".

Para el desarrollo de la estrategia en el departamento de Caldas se ha estructurado un modelo operativo, en el cual se proponen diferentes elementos que parten del análisis de los indicadores en salud de cada municipio, y ha logrado una articulación no sólo entre profesionales, 
sino entre actores y sectores. En este mismo sentido, tal como lo enuncia Sierra ${ }^{20}$, cada municipio debe trabajar de acuerdo con el Análisis de la Situación de Salud para intervenir los determinantes sociales, estimular el trabajo en equipo y en red, fortalecer los espacios comunitarios y sociales, integrar las acciones locales de salud, educación, bienestar social, cultura, entre otros. Esta profundidad en el análisis permite conocer las dinámicas locales, que como se mencionó anteriormente orientan la toma de decisiones en contexto ${ }^{23}$.

Para la implementación de la APS, la vinculación de la academia fue valorada como un elemento importante para el desarrollo del modelo, lo anterior permite una visión colaborativa y concepción interdisciplinar en la priorización, análisis e intervención de diferentes problemas, tal como lo plantean Pulcini y Lang ${ }^{24}$.

El modelo operativo para la dinamización de la APS en el departamento de Caldas podría ser retomado a nivel nacional para la Política "Modelo de Acción Integral Territorial" (MAITE), lo anterior en cumplimiento a los mandatos internacionales que invitan a los países a crear o fortalecer sistemas integrales e integrados de salud con enfoque de Atención Primaria en Salud ${ }^{25}$.

Como parte del modelo operativo y elemento innovador de la estrategia, se identificó la necesidad de trabajar un modelo de TeleAPS, esto concuerda con los resultados de Tasca et al. ${ }^{18}$, quienes concluyen que, para el fortalecimiento de la APS en Brasil, es necesaria la innovación en materia de tecnologías asistenciales, de gestión y de comunicación, siempre teniendo como eje a los actores.

En el trabajo comunitario se pudo evidenciar la valoración que se realiza del modelo de Atención Primaria Social, visto por los actores como una estrategia que ha posibilitado un abordaje "desde y para la comunidad", es así como en la gran mayoría de los municipios se informó que antes de su implementación se trataba a la persona únicamente desde lo biológico y posteriormente se realiza un análisis integral, incluyendo el contexto de vida, para intervenir sus riesgos con el compromiso comunitario. En este sentido, trabajos como el de Domínguez et al. ${ }^{26}$ valoran la importancia de la Atención Primaria para un proyecto denominado "Familias + activas". Otro aspecto relevante que emergió del estudio es la importancia del compromiso político y la inclusión en las agendas de gobierno de la estrategia como la posibilidad de sostenibilidad en el tiempo, aspecto sobre el cual hace un llamado la OMS en su documento ${ }^{27}$. En este mismo sentido, el trabajo de Egaña concluye que este tipo de estrategias que fomentan la participación de la comunidad aportan a la calidad de vida de los colectivos ${ }^{28}$.

\section{Conclusiones}

A manera de cierre, a lo largo del estudio se denota la importancia de la articulación de la academia, estado y sociedad civil, sin solapar el compromiso social y la articulación de actores sociales y comunitarios, entre otros anclados a sus territorios, así como sectores públicos y privados, bajo la invitación de Almeida en $2018^{17}$ de relanzar la estrategia de APS a 40 años de la Declaración de Alma-Ata, aunque con una mirada desde lo social y de lo comunitario si se quiere. Se denota que los indicadores en salud para el departamento de Caldas han mejorado a partir de la implementación de la estrategia de Atención Primaria Social, este trabajo confirma lo encontrado por otros actores colombianos, quienes proponen la necesidad de que a nivel nacional se unifiquen criterios en torno a APS para el logro de objetivos comunes en beneficio del sistema mismo ${ }^{29}$.

Financiación: el presente estudio contó con la cooperación de la Organización Panamericana de la Salud.

\section{Referencias}

1. Organización Mundial de la Salud. Atención Primaria de Salud. Informe de la Conferencia Internacional sobre Atención Primaria de Salud Alma-Ata, URSS, 6-12 de septiembre de 1978. Ginebra: Organización Mundial de la Salud; 1978; p. 1-3.

2. Cofiño R, Aviñó D, Benedé CB, Botello B, Cubillo J, Morgan A, et al. Promoción de la salud basada en activos: ¿cómo trabajar con esta perspectiva en intervenciones locales? Gac Sanit. 2016; 30: 93-98. doi: https://doi.org/10.1016/j. gaceta.2016.06.004

3. Ley 1438 de 2011. Por medio de la cual se reforma el Sistema General de Seguridad Social en Salud y se dictan otras disposiciones. Bogotá: Congreso de la República de Colombia, 2011.

4. Resolución número 2626 de 2019. Por la cual se modifica la Política de Atención Integral en Salud -PAIS y se adopta el Modelo de Acción Integral Territorial-MAITE. Bogotá: Ministerio de Salud y Protección Social, 2019.

5. Franco-Giraldo A. El rol de los profesionales de la salud en la atención primaria en salud (APS). Rev Fac Nac Salud Pública. 2015; 33(3): 414-424. doi: 
https://doi.org/10.17533/udea.rfnsp.v33n3a11

6. Plan de Desarrollo Departamental 2016-2019. Caldas, territorio de oportunidades. Manizales, Gobernación de Caldas, 2016; p. 1-2.

7. Seguimiento al sector salud en Colombia, Así Vamos en Salud. Informe Anual 2019, Perspectiva del Sistema de Salud Colombiano. Colección: Tendencias de la Salud en Colombia. Bogotá: Así Vamos en Salud, 2019; p. 1-119.

8. Organización Panamericana de la Salud. Bogotá: Organización Panamericana de la Salud; c2022. El legado en Atención Primaria Social que Caldas le entrega al mundo.

9. Pérez SL, Arrivillaga M. Redes integradas de servicios de salud en el marco de la atención primaria en salud en países seleccionados de América Latina. Salut Sci Spiritus. 2017; 3(2): 32-49

10. Acosta LA. Guía práctica para la sistematización de proyectos y programas de cooperación técnica. Santiago (Chile): Oficina Regional de la FAO para América Latina y El Caribe. 2005.

11. Campo JO. Política de atención integral en salud (PAIS), "hacia mejores condiciones de la salud" en Colombia. Vestig Ire. 2020; 14(1): 77-91.

12. Organización de las Naciones Unidas para la Alimentación y la Agricultura (FAO). Buenas prácticas en la FAO: Sistematización de experiencias para el aprendizaje continuo. Roma: Organización de las Naciones Unidas para la Alimentación y la Agricultura. 2013; p. 1-12.

13. Organización de las Naciones Unidas para la Agricultura y la Alimentación (FAO). Guía Metodológica de Sistematización. Programa Especial para la Seguridad Alimentaria PESA en Centroamérica. Roma: Organización de las Naciones Unidas para la Agricultura y la Alimentación (FAO). 2004.

14. Resolución 8430 de 1993. Por la cual se establecen las normas científicas, técnicas y administrativas para la investigación en salud. Bogotá: Ministerio de Salud y Protección Social, 1993.

15. Ley 100 de 1993. Por la cual se crea el sistema de seguridad social integral y se dictan otras disposiciones. Bogotá: Congreso de la República de Colombia, 1993.

16. Ministerio de Salud y Protección Social, Instituto Proinapsa-UIS. La ruta de la educación y comunicación para la salud. Orientaciones para su aplicación estratégica. Bucaramanga: Instituto Proinapsa, 2014; p. 136.

17. Almeida G, Artaza O, Donoso N, Fábrega R. La atención primaria de salud en la Región de las Américas a 40 años de la Declaración de Alma-
Ata. Rev Panam Salud Pública. 2018; 42: e104. doi: https://doi.org/10.26633/RPSP.2018.104

18. Tasca R, Massuda A, Carvalho WM, Buchweitz C, Harzheim E. Recomendações para o fortalecimento da atenção primária à saúde no Brasil. Rev Panam Salud Publica. 2020; 44: e4. doi: https://doi. org/10.26633/RPSP.2020.4

19. Osorio AG, Álvarez CV, Rubio AP. Calidad de los instrumentos que valoran las funciones de la atención primaria: revisión sistemática. Hacia Promoc. Salud. 2018; 25(1):1 09-29. doi: https:// doi.org/10.17151/hpsal.2020.25.1.8

20. Sierra-Forero LE. La estrategia de atención primaria en salud desde la experiencia de enfermería en el departamento de Boyacá. Rastrosyrostros. 2019; 4(6): 26-38.

21. Suárez R, Conté $\mathrm{G}$, Olarte $\mathrm{M}$, Zamora S. Sistematización de experiencias desarrolladas en el país sobre APS y RISS en los niveles territoriales. Bogotá: Instituto Nacional de Salud, 2012; p. 122.

22. Rodríguez-Villamizar LA, Ruiz-Rodríguez $\mathrm{M}$, Acosta-Ramírez N. Evaluación de un modelo de atención primaria en salud en Santander, Colombia. Rev Fac Nac Salud Pública. 2016; 34(1): 88-95. doi: https://doi.org/10.17533/udea.rfnsp.v34n1a11

23. Yepes CE, Marín YA. Desafíos del análisis de la situación de salud en Colombia. Biomédica. 2018; 38(2): 162-72. doi: https://doi.org/10.7705/ biomedica.v38i0.3594

24. Pulcini JA, Lang CS. Estratégias para aprimorar a profi ssão da enfermagem como parceira-chave para melhorar a atenção primária em todo o hemisfério. Acta Paul Enferm. 2018; 31(3): III-VI. doi: https:// doi.org/10.1590/1982-0194201800033

25. Pedraza CC, Pagano JP, Pescetto C, Prieto L. Espacio fiscal para el financiamiento sostenible de los sistemas de salud y la salud universal. Rev Panam Salud Pública. 2018; 42: e197. doi: https:// doi.org/0.26633/RPSP.2018.197

26. Domínguez-Fernández S, Valdivieso-Fernández de Valderrama B, Martínez-Ortega RM, Aznar-Laín S, Romero-Blanco C, Villalvilla-Soria DJ. Familias + Activas: Enfermería familiar y comunitaria ante el reto de la atención a la Obesidad Infantil en Atención Primaria. RqR Enfermería Comunitaria. 2019; 7(3): 28-40.

27. Organización Mundial de la Salud (OMS). Conferencia Mundial sobre Atención Primaria de Salud. Desde Alma-Ata hacia la cobertura sanitaria universal y los Objetivos de Desarrollo Sostenible. Astaná (Kazajstán), 25 y 26 de octubre de 2018. Ginebra: Organización Mundial de la Salud, 2018; p. 6. 
28. Egaña Rojas D, Iglesias Vejar L, Cerda Rioseco R, Molina Carrasco P, Gálvez Espinoza P. Participación social en la atención primaria en salud: tensiones y contradicciones. Aten Primaria. 2020; 52(10): 690-696. doi: https://doi.org/10.1016/j. aprim.2020.02.013

29. González Quiñones JC. Resultados, sistematización, análisis y recomendaciones de la evaluación de las experiencias de atención primaria en Colombia. Cart Comunitaria. 2018; 23(132): 13-29. 